\title{
Autologous FRa-4SCAR-expressing T-cells 4SCAR-FRa
}

National Cancer Institute

\section{Source}

National Cancer Institute. Autologous FRa-4SCAR-expressing T-cells 4SCAR-FRa. NCI

Thesaurus. Code C150698.

A preparation of genetically modified autologous T-cells transduced with a replication incompetent, self-inactivating lentiviral vector expressing a fourth generation chimeric antigen receptor (4SCAR) consisting of an anti-folate receptor alpha (FRa; folate receptor 1; FOLR1) sing le chain variable fragment (scFv) that is coupled to the costimulatory signaling domains CD28, CD137, CD27 and the zeta chain of the T-cell receptor (CD3zeta; CD3z), and is fused with the suicide gene inducible caspase 9 (iCasp9), with potential immunostimulating and antineoplastic activities. Upon administration, autologous FRa-4SCAR-expressing T-cells 4SCAR-FRa are directed to and induce selective toxicity in FRa-expressing tumor cells. iCasp9 consists of a human FK506 drugbinding domain with an F36V mutation (FKBP12-F36V) linked to human caspase 9. If the administered T -cells lead to unacceptable side effects, the chemical homodimerizer AP1903 can be administered. AP1903 binds to the drug binding FKBP12-F36V domain and induces activation of caspase 9, which results in the apoptosis of the administered T-cells and enhances safety of this agent. FRa is overexpressed in various tumor cell types, and is associated with increased leukemic cell proliferation and aggressiveness. CD28, CD137 and CD27, T-cell surface-associated co-stimulatory molecules, are required for full T-cell activation and enhance both proliferation of T-cells and antitumor activity. 